\title{
ON ONE SIDED IDEALS OF A PRIME TYPE
}

\author{
KWANGIL KOH
}

ABSTRACT. The notion of prime type for right ideals is analogous to that of prime ideal in a commutative ring, and the right dimension of the ring $R$ is defined using chains of right ideals of prime type. The meaning of zero right dimension, finite dimension for right primitive rings, and related topics are studied.

1. Introduction. N. H. McCoy has defined that a two sided ideal $S$ in a ring $R$ is a prime ideal provided that if $A B \subseteq S, A, B$ are right ideals, then either $A \subseteq S$ or $B \subseteq S$ (see [5]). Let us define that a right (left) ideal $I$ in a ring $R$ is of a prime type if and only if $I \neq R$ and $A B \subseteq I, A, B$ are right (left) ideals, implies that either $A \subseteq I$ or $B \subseteq I$. It is easy to see that if $R$ is a commutative ring then an ideal of a prime type is just a prime ideal. By a chain of right ideals of prime type in a ring $R$ we mean a finite strictly increasing sequence $P_{0} \subset P_{1}$ $\subset \cdots \subset P_{n}$; the length of the chain is $n$. We define the right dimension of a ring $R$, which is denoted by $\operatorname{dim}_{r} R$, to be the supremum of the lengths of all chains of right ideals of a prime type in $R$ : it is an integer $\geqq 0$, or $\infty$. The left dimension of $R$, which is denoted by $\operatorname{dim}_{l} R$, is similarly defined. The main results in this paper are:

(a) $\operatorname{dim}_{r} R=0$ if and only if $R$ modulo the prime radical is a strongly regular ring.

(b) $\operatorname{dim}_{r} R=0$ and $R$ is a right noetherian ring if and only if $R$ is a right artinian ring and every right ideal of a prime type is a left ideal.

(c) If $R$ is a (right) primitive ring then $\operatorname{dim}_{r} R=n$ if and only if $R$. is isomorphic to the $n+1 \times n+1$ matrix ring over a division ring.

(d) Every right ideal of a prime type in a ring $R$ is a left ideal if and only if every right ideal of a prime type is almost maximal (refer [3] for definition).

In the sequel, unless otherwise stated, $R$ will always denote a ring with 1 and if $T$ is a nonempty subset of a (right) $R$-module $M$ then

$$
T^{\perp}=\{r \in R \mid t r=0 \text { for all } t \in T\} .
$$

2. If $R$ is a ring let $p(R)$ be the set of right ideals of a prime type and $m(R)$ be the set of maximal right ideals. Then $m(R) \subseteq p(R)$. For

Received by the editors March 13, 1970.

AMS 1969 subject classifications. Primary 1620, 1625, 1640.

Key words and phrases. Normalizer, injective hull, quasi-injective hull, noetherian, artinian, strongly regular ring. 
if $I \in m(R)$ and $A B \subseteq I$ for some right ideals $A$ and $B$ such that $A \nsubseteq I$ then $A+I=R$ and $R B=(A+I) B \subseteq I$ and $B \subseteq I$.

2.1. Proposition. If $I \in p(R)$ then the largest two sided ideal of $R$, $s(I)$, which is contained in $I$ is a member of $p(R)$.

Proof. $s(I)=\{r \in R \mid R r \subseteq I\}$ and it is the largest two sided ideal of $R$ which is contained in $I$. Let $A B \subseteq s(I)$ for some right ideals $A$ and $B$. Then $R A B \subseteq R s(I) \subseteq s(I) \subseteq I$ and either $R A \subseteq I$ or $R B \subseteq I$. In any case, either $A \subseteq s(I)$ or $B \subseteq s(I)$. Thus $s(I) \in p(R)$.

2.2. CoRollary. The prime radical of $R, \operatorname{rad} R$, is equal to $\bigcap_{I_{\alpha} \in p(R)} I_{\alpha}$.

Proof. $\bigcap_{I_{\alpha} \in p(R)} I_{\alpha} \subseteq \operatorname{rad} R \subseteq \bigcap_{I_{\alpha} \in p(R)} s\left(I_{\alpha}\right) \subseteq \bigcap_{I_{\alpha} \in p(R)} I_{\alpha}$.

2.3. Lemma. If $p(R) \subseteq m(R)$ then every maximal right ideal of $R$ is also a left ideal.

Proof. If $I \in m(R)$ then $I \in p(R)$. Hence by $2.1, s(I) \in p(R)$ and $s(I) \in m(R)$ by hypothesis. Since $s(I) \subseteq I, s(I)=I$.

2.4. Lemma. If $p(R) \subseteq m(R)$ then every nilpotent element of $R$ is contained in $\operatorname{rad} R$.

Proof. Let $x \in R$ such that $x^{n}=0$ for some positive integer $n$. If $x \notin \operatorname{rad} R$ then there exists $I \in p(R)$ such that $x \notin I$. Since $I$ is also a left ideal by $2.3, R / I$ is a division ring. Hence $x+I$ is not a nilpotent element. This is impossible.

2.5. LeMma. Let $R^{\prime}=R / \mathrm{rad} R$. If $p(R) \subseteq m(R)$ then for any $b \in R^{\prime}$, $b r=0$ if and only if $r b=0$ for any $r \in R^{\prime}$ and $(b)^{\perp}$ is a two sided ideal.

PRoof. We first note that if $p(R) \subseteq m(R)$, by 2.4 , there is no nonzero nilpotent element in $R^{\prime}$. Let $b \in R^{\prime}$. If $b r=0$ for some $r \in R^{\prime}$ then $(r b)(r b)=0$. Hence $r b=0$. Similarly if $r b=0$ for some $r \in R^{\prime}$ then $(b r)(b r)=0$ and $b r=0$. Therefore $(b)^{\perp}$ is a two sided ideal.

2.6. Lemma. Let $R^{\prime}=R / \mathrm{rad} R$. For each $I \in p\left(R^{\prime}\right)$, define $O_{I}$ $=\left\{a \in R^{\prime} \mid a x=0\right.$ for some $\left.x \notin I\right\}$. If $p(R) \subseteq m(R)$ then $O_{I}=I$.

Proof. If $p(R) \subseteq m(R)$ then clearly $p\left(R^{\prime}\right)=m\left(R^{\prime}\right)$ and $I$ is a two sided ideal by 2.3. Hence if $x \notin I$ and $y \notin I$ then $x \cdot y \notin I$, since $R^{\prime} / I$ is a division ring. Hence $O_{I} \subseteq I$. Suppose there is $a \in I$ such that $a \notin O_{I}$. Let $\Gamma=\left\{x \in R^{\prime} \mid x \notin I\right\}$. Then $\Gamma$ is closed under multiplication. Let $\Gamma_{0}$ be the multiplicative system which is generated by $\Gamma \cup\left\{a, a^{2}, a^{3}, \cdots\right\}$. If $t \in \Gamma_{0}$ then $t=a^{i_{0}} x_{0} a^{i_{1}} x_{1} a^{i_{2} x_{2}} \cdots a^{i_{n}} x_{n} a^{i_{n+1}}$ for some positive integer $n$ where $x_{j} \in \Gamma, j=0,1,2, \cdots, n, i_{k}$ are nonnegative integers, $k=0,1, \cdots, n+1$, and $a^{0}=1$. First we show that for any $x \in \Gamma, a^{i} x \neq 0$ for any positive integer $i$. Suppose $a^{i} x=0$ for 
some positive integer. Let $i$ be the least such integer. Then $a a^{i-1} x=0$ for some positive integer. Let $i$ be the least such integer. Then $a a^{i-1} x=0$ and $a^{i-1} x \neq 0$ and $a^{i-1} x a=0$ by 2.5 . Thus $a^{i-1} x a^{i-1} x=0$ and $a^{i-1} x=0$ by 2.4. This is impossible. Now we will show that $t \neq 0$. Suppose $0=t=a^{i_{0} x_{0}} a^{i_{1} x_{1}} a^{i_{2} x_{2}} \cdots a^{i_{n}} x_{n} a^{i_{n+1}}$. Let $i=i_{0}+i_{1}+\cdots+i_{n+1}$. Since $a^{i_{1}} x_{1} a^{i_{2}} x_{2} \cdots a^{i_{n} x_{n}} a^{i_{n+1}} \in\left(a^{i_{0}} x_{0}\right)^{\perp}$ and $\left(a^{i_{0}} x_{0}\right)^{\perp}$ is a left ideal by 2.5, $\quad\left(a^{i_{0} x_{0}}\right)\left(x_{1} x_{2} \cdots x_{n}\right) a^{i-i_{1}} a^{i_{1}} x_{1} a^{i_{2} x_{2}} \cdots a^{i_{n}} x_{n} a^{i_{n+1}}=0 . \quad$ Since

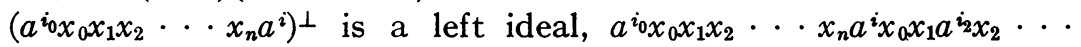

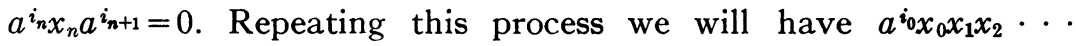
$x_{n} a^{i} x_{0} x_{1} x_{2} \cdots x_{n} a^{i} \cdots a^{i} x_{0} x_{1} x_{2} \cdots x_{n} a^{i_{n+1}}=0$. Thus we will have

$$
\begin{gathered}
\left(a^{i} x_{0} x_{1} \cdots x_{n}\right)\left(a^{i} x_{0} x_{1} \cdots x_{n}\right) \cdots\left(a^{i} x_{0} x_{1} \cdots x_{n}\right)=0 . \\
(n+1 \text { times })
\end{gathered}
$$

This means that $a^{i} y=0$ for some $y=x_{0} x_{1} \cdots x_{n} \in \Gamma$ and we arrive at a contradiction. Now let $S$ be a two sided ideal of $R^{\prime}$ which is maximal with respect to a property that $S \cap \Gamma_{0}=\varnothing$. Then $S$ is a prime ideal of $R^{\prime}$, which is properly contained in $I$. This is a contradiction. Thus $O_{I}=I$.

2.7. Lemma. Assume $p(R) \subseteq m(R)$. For any $I \in p\left(R^{\prime}\right),\left(R^{\prime} / I\right)^{\wedge}$ $=R^{\prime} / I$.

Proof. Let $0 \neq x \in\left(R^{\prime} / I\right)^{\wedge}$. If $x I \neq 0$ then $x I \supseteq R^{\prime} / I$ since $R^{\prime} / I$ is simple. Hence $1+I=x a$ for some $a \in I$. By Lemma 2.6, $a y=0$ for some $y \notin I$. Therefore, $0 \neq(1+I) y=x a y=0$, a contradiction. Thus $x^{\perp}=I$ and $x R^{\prime} \cong R^{\prime} / x^{\perp}$ is simple. Thus $\left(R^{\prime} / I\right)^{\wedge}=R^{\prime} / I$.

2.8. TheOREM (VIllamayor). Every simple $R$-module is injective if and only if every right ideal of $R$ is the intersection of maximal right ideals.

Proof. Let $I$ be a right ideal of $R$ and let $\left\{I_{\alpha} \mid \alpha \in \Lambda\right\}$ be the family of maximal right ideals of $R$ which contain $I$. Then $I \subseteq \bigcap_{\alpha \in \Delta} I_{\alpha}$. Suppose $x \in \bigcap_{\alpha \in \Delta} I_{\alpha}$ but $x \notin I$. Then define $\Sigma_{x}=\{J \mid J$ is a right ideal, $I \subseteq J \neq R$ and $x \notin J\}$. By Zorn's lemma, $\Sigma_{x}$ has a maximal member, say $J^{*}$. It is clear that $x R+J^{*} / J^{*}$ is a simple $R$-module. Since $x R+J^{*} / J^{*}$ is a submodule of $R / J^{*}$ and every simple module is injective, $R / J^{*}=x R+J^{*} / J^{*} \oplus K / J^{*}$ for some right ideal $K$. If $K \neq J^{*}$ then $x$ would be an element of $K$. Hence $K=J^{*}$ and $x R+J^{*}=R$. Now let $J_{1}$ be a maximal right ideal of $R$ such that $J^{*} \subseteq J_{1}$. Then $J_{1}=I_{\alpha}$ for some $\alpha \in \Lambda$ since $I \subseteq J^{*} \subseteq J_{1}$ and $x \in J_{1}$. This means that $J_{1}=R$. This is a contradiction. To prove the converse, assume that every right ideal of $I$ is the intersection of maximal right ideals. Let $M$ be a simple $R$-module. Let $0 \neq x \in \hat{M}$. Then $(x)^{\perp}=\bigcap_{\alpha \in \Lambda} I_{\alpha}$ where 
$\left\{I_{\alpha} \mid \alpha \in \Lambda\right\}$ is a set of maximal right ideals. Let $M_{\alpha}=R / I_{\alpha}$. Then $M_{\alpha}$ is a simple $R$-module and $x R$ is a subdirect sum of $\left\{M_{\alpha}\right\}_{\alpha \in \Delta}$. Let $x R \stackrel{\mu}{\rightarrow} \prod M_{\alpha} \stackrel{p_{\alpha}}{\rightarrow} M_{\alpha}$ where $\mu$ is an injection and $p_{\alpha}$ is a projection for each $\alpha \in \Lambda$. $p_{\alpha} \circ \mu$ is an epimorphism for each $\alpha \in \Lambda$. If there is $\alpha \in \Lambda$ such that $p_{\alpha} \circ \mu$ is a monomorphism then $x R \equiv M_{\alpha}$ and $x R$ would be simple. Hence $x R=M$ and $M=\hat{M}$. So suppose $p_{\alpha} \circ \mu$ is not a monomorphism for all $\alpha \in \Lambda$. Then the kernel of $p_{\alpha} \circ \mu$ must contain $M$ since $x R$ is a large submodule of $\hat{M}$. Thus $\bigcap_{\alpha \in \Delta} \operatorname{ker}\left(p_{\alpha} \circ \mu\right)$. Of course, this is impossible.

2.9. THEOREM. The following statements are equivalent:

(a) $\operatorname{dim}_{r} R=0$.

(b) $\operatorname{dim}_{l} R=0$.

(c) $R / \mathrm{rad} r$ is a strongly regular ring (i.e. for any a there is $x$ such that $\left.a=a x a=a^{2} x\right)$.

Proof. First we note that if $R / \mathrm{rad} R$ is a strongly regular ring then every principal right ideal of $R / \mathrm{rad} R$ is generated by a central idempotent and every principal left ideal of $R / \operatorname{rad} R$ is also generated by a central idempotent. Hence every right ideal of $R / \mathrm{rad} R$ is a left ideal and every left ideal of $R / \operatorname{rad} R$ is a right ideal. Let $P$ be a right ideal of $R$ which is of prime type. Then $P / \operatorname{rad} R$ is a right ideal. Let $P$ be a right ideal of $R$ which is of a prime type. Then $P / \operatorname{rad} R$ is a right ideal of $R / \operatorname{rad} R$ which is of a prime type, hence it is a two sided ideal. Now $(R / \operatorname{rad} R) /(P / \operatorname{rad} R)$ is a prime ring which is strongly regular. Since a strongly regular ring contains no nonzero nilpotent element, $R / P$ must be an integral domain which is a regular ring. Hence $R / P$ must be a division ring. Thus $P$ is a maximal right ideal of $R$. Similarly if $P$ is a left ideal of $R$ which is of a prime type then $P$ is a maximal left ideal. Hence the right $\operatorname{dim} R=0=$ the left $\operatorname{dim} R$. Now assume (a). Let $R^{\prime}=R / \mathrm{rad} R$. Then by 2.7 , every simple $R^{\prime}$-module is injective. Hence by 2.8 , every right ideal of $R^{\prime}$ is the intersection of maximal right ideals. In particular, if $a$ is a nonzero element of $R^{\prime}$, then $a^{2} R^{\prime}=\bigcap_{\alpha \in \Lambda} I_{\alpha}$ where $I_{\alpha}, \alpha \in \Lambda$, is a maximal right ideal which contains $a^{2} R^{\prime}$. If $a \notin a^{2} R^{\prime}$ then $a \notin I_{\alpha}$ for some $\alpha \in \Lambda$ and $a^{2} \in I_{\alpha}$. This is impossible since $I_{\alpha}$ is also a left ideal by 2.3 and $R^{\prime} / I_{\alpha}$ is a division ring. This proves that $a=a^{2} x$ for some $x \in R$. Since $(a x a-a)^{2}=0$, and $R^{\prime}$ has no nilpotent element except $0, a=a x a=a^{2} x$.

2.10. Lemma. If $I \in p(R)$ then $(I: x)=\{r \in R \mid x r \in I\} \in p(R)$ for any $x \in R$.

Proof. Let $A B \subseteq(I: x)$ where $A, B$ are right ideals. Then $x A B \subseteq I$. Since $x A$ is also a right ideal and $I \in p(R)$, this means that either 
$x A \subseteq I$ or $R B \subseteq I$. In any case, either $A \subseteq(I: x)$ or $B \subseteq(I: x)$.

2.11. Lemma. If $R_{1}$ and $R_{2}$ are two rings and $f$ is a ring homomorphism of $R_{\mathbf{1}}$ onto $R_{\mathbf{2}}$, then $f^{-1}(I) \in p\left(R_{\mathbf{1}}\right)$ for any $I \in p\left(R_{\mathbf{2}}\right)$.

Proof. Straightforward.

2.12. THEOREM. $\operatorname{dim}_{r} R=0$ and $R$ is a right noetherian ring if and only if $R$ is a right artinian ring and every right ideal of a prime type is a left ideal.

Proof. Suppose $\operatorname{dim}_{r} R=0$ and $R$ is a right noetherian ring. By 2.9, $R / \operatorname{rad} R$ is a right noetherian ring which is regular. Hence $R / \operatorname{rad} R$ is a right artinian ring (refer, for example, [4]). Since $R / \mathrm{rad} R$ is semisimple, the Jacobson radical $J(R)=\operatorname{rad} R$ and it is nilpotent since $R$ is right noetherian. Hence by Theorem (Chase) of [1, p. 189], $R$ is a right artinian ring. If $I$ is a right ideal of a prime type then $I \supseteq \operatorname{rad} R$ by 2.2 and $I / \mathrm{rad} R$ is a right ideal of $R / \mathrm{rad} R$. Since every right ideal of $R / \mathrm{rad} R$ is also a left ideal, $I / \mathrm{rad} R$ is a left ideal of $R / \mathrm{rad} R$ and this means that $I$ is a left ideal in $R$. Conversely, assume $R$ is right artinian and every right ideal of a prime type is a left ideal. Let $I$ be a right ideal of a prime type. Since $I$ is also a left ideal, $I$ is a prime ideal and $R / I$ is a right artinian simple ring. Now if $x \in R, x \notin I$ then Ann $(x+I)=\{y+I \mid y \in R$ and $x y \in I\}$ is of a prime type. Hence by 2.11 the set $\{y \in R \mid x y \in I\}$ is a right ideal of a prime type in $R$ and it is a two sided ideal by hypothesis. Thus Ann $(x+I)$ is a two sided ideal in $R / I$ and it must be a zero ideal since $R / I$ is a simple ring. Hence $R / I$ is an artinian integral domain and $I$ is a maximal right ideal which is also a left ideal. Thus $\operatorname{dim}_{r} R=0$. Any right artinian ring is also a right noetherian ring and this will conclude the proof.

3. If $I$ is a right ideal of a ring $R$, the set $N(I)=\{x \in R \mid x I \subset I\}$ is called the "normalizer of $I$ " in $R$ (refer $\left[2\right.$, p. 25]). Let $N^{*}(I)$ $=\{x \in N(I) \mid x y \in I$ if and only if $y \in I\}$. Let $R \backslash I$ denote the (set) complement of $I$ in $R$. In [5], we have defined that a right ideal $I$ of a ring $R$ is almost maximal if and only if:

(A1) for any $a, b \in R \backslash I$, there exist $r_{1}, r_{2} \in R$ and $c \in N^{*}(I)$ such that $a r_{1} \equiv b r_{2} \equiv c \bmod I$.

(A2) If $a \in R \backslash I$ then either $a \in N(I)$ or $a r \equiv a i \bmod I$ for some $r \in R \backslash I$ and $i \in I$.

It is also proven in [3] that a right ideal $I$ of a ring $R$ is almost maximal if and only if the endomorphism ring of the quasi-injective hull of the right $R$-module $R / I$ is a division ring and every nonzero 
submodule of $R / I$ contains an isomorphic image of $R / I$. In this section, we will prove that every right ideal of a prime type is a left ideal if and only if every right ideal of a prime type is almost maximal.

3.1. Lemma. $I \in p(R)$ if and only if, for any nonzero submodule $N$ of $R / I, N x=0$ if and only if $(R / I) x=0$.

Proof. Suppose $I \in p(R)$ and let $N=A / I$ for some right ideal $A$ such that $A \neq I$. If $N x=0$ for some $x \in R$ then $A x \subseteq I$ and the set $B=(I: A)=\{x \in R \mid A x \subseteq I\}$ is a right ideal of $R$ and $A B \subseteq I$. Since $A \Phi I, R B \subseteq I$ and $N^{\perp}=(R / I)^{\perp}$. Conversely assume that $N^{\perp}=(R / I)^{\perp}$. Let $A B \subseteq I$ for some right ideals $A$ and $B$ in $R$. If $A \Phi I$, let $N=A+I / I$. Then $N$ is a nonzero submodule of $R / I$ and $N^{\perp} \supseteq B$. Hence $(R / I)^{\perp} \supseteq B$.

3.2. Lemma. Let $R$ be a prime ring and let $\Gamma=\{I \in p(R) \mid s(I)=0\}$. If $\Lambda$ is a nonempty subset of $\Gamma$ then $\bigcap_{I_{\alpha} \in \Lambda} I_{\alpha}$ is a member of $\Gamma$.

Proof. Let $A B \subseteq \cap_{I_{\alpha} \in \Lambda} I_{\alpha}$ for some right ideals $A$ and $B$. If $A \nsubseteq \cap_{I_{\alpha} \in \Lambda} I_{\alpha}$, then there is $I_{\alpha} \in \Lambda$ such that $A \nsubseteq I_{\alpha}$. Since $A B \subseteq I_{\alpha}$ and $I_{\alpha} \in p(R), R B \subseteq I_{\alpha}$. Since $s\left(I_{\alpha}\right)=0$, this means $R B=0$ and $B=0$. Thus $B \subseteq \bigcap_{I_{\alpha} \in \Lambda} I_{\alpha}$. This proves that $\bigcap_{I_{\alpha} \in \Lambda} I_{\alpha} \in \Gamma$.

3.3. Lemma. Suppose that if $I \in p(R)$ then $I$ is almost maximal. Then $I=s(I)$ for each $I \in p(R)$.

Proof. Let $I \in p(R)$ and $M=R / I$. Then $M$ is a faithful $R / s(I)$ module and $R / s(I)$ is a prime ring since $s(I) \in p(R)$ by 2.1. If $m \in M$, let Ann $(m)=\{r+s(I) \in R / s(I) \mid m(r+s(I))=0\}$. If $m \neq 0$ then Ann $(m) \in p(R / s(I))$ by 2.10 since $I / s(I) \in p(R / s(I))$. Consider $B=s($ Ann $(m))$, the largest two sided ideal of $R / s(I)$ which is contained in Ann (m). If $B$ is not zero then the set $N=\{m \in M \mid m B=0\}$ is a nonzero submodule of $M$. Since $N$ contains an isomorphic image of $M, N B=0$ implies that $M B=0$. Since $M$ is faithful, this means that $B$ is zero of $R / s(I)$. This is a contradiction. Thus $s($ Ann $(m))$ is zero. Now let $m_{1}, m_{2}$ be nonzero elements of $M$. Then Ann $\left(m_{1}\right)$ $\cap$ Ann $\left(m_{2}\right) \in p(R / s(I))$ by 3.2. Let $\pi$ be the canonical mapping of $R$ onto $R / s(I)$. Then

$$
\pi^{-1}\left(\operatorname{Ann}\left(m_{1}\right) \cap \operatorname{Ann}\left(m_{2}\right)\right)=\left(m_{1}\right)^{\perp} \cap\left(m_{2}\right)^{\perp} \in p(R)
$$

by 2.11 . Hence $\left(m_{1}\right)^{\perp} \cap\left(m_{2}\right)^{\perp}$ is an almost maximal right ideal. Since every nonzero submodule of $R /\left(m_{1}\right)^{\perp} \cap\left(m_{2}\right)^{\perp}$ is large, $\left(m_{1}\right)^{\perp} \cap\left(m_{2}\right)^{\perp}$ $=\left(m_{1}\right)$ or $\left(m_{1}\right)^{\perp} \cap\left(m_{2}\right)^{\perp}=\left(m_{2}\right)^{\perp}$. In any case, either $\left(m_{1}\right)^{\perp} \subseteq\left(m_{2}\right)^{\perp}$ or $\left(m_{2}\right)^{\perp} \subseteq\left(m_{1}\right)^{\perp}$. In other words, given $a_{1} \notin I, a_{2} \notin I$, either $\left(I: a_{1}\right)$ $\subseteq\left(I: a_{2}\right)$ or $\left(I: a_{2}\right) \subseteq\left(I: a_{1}\right)$. Now suppose $a I \Phi I$ for some $a \in R$ (hence $a \notin I)$. Then (I:a) $I I$ and therefore, by Lemma 2.0 (iii) of [3], 
$(I: a) \Phi I$. However, by Lemma 2.0 (ii) of [3], there is $r \in R$ such that $(I: a r)=I$, with $\operatorname{ar} \notin I$. This is a contradiction.

3.4. CoROllary. If $R$ is a (right) primitive ring and every right ideal of a prime type is almost maximal then $R$ is a division ring.

Proof. Let $I$ be a maximal right ideal such that $s(I)=0$. Since $I \in p(R)$, by 3.3, $0=s(I)=I$. Hence $R$ is a division ring.

3.5. Lemma. Let $R$ be a prime ring. If $C$ is a complement right ideal of $R$ then $C \in p(R)$.

Proof. If $C$ is a complement right ideal then there exists a right ideal $J$ such that $J \cap C=(0)$ and if $I$ is a right ideal such that $I \supset C$ and $I \neq C$ then $J \cap I \neq(0)$. Let $A B \subseteq C$ for some right ideals $A$ and $B$ in $R$. If $A \Phi C$ then $A+C \supset C$ and $A+C \neq C$. Hence $(A+C) \cap J \neq 0$. Let $j=a+c \neq 0$ for some $j \in J, a \in A$ and $c \in C$. Then $j R B \subseteq C \cap J$ and $R B \subseteq(j)^{\perp}$. Since $R$ is a prime ring, this means $B=0 \subseteq \mathrm{C}$.

3.6. THEOREM. Every right ideal of a prime type in a ring $R$ is almost maximal if and only if every right ideal of a prime type is a left ideal.

Proof. Suppose that if $I \in p(R)$ then $I$ is almost maximal. Then by 3.3, $I=s(I)$. Thus $I$ is also a left ideal. Conversely, assume that every right ideal of a prime type is a left ideal. Then for each $I \in p(R)$, $R / I$ is a prime ring and it must be an integral domain since $(I: a)$ $\in p(R)$ for each $a \in R \backslash I$ by 2.10 and only two sided annihilator ideals in a prime ring are entire ring and zero ideal. By 3.5 , if $\bar{C}$ is a complement right ideal of $R / I$ then $\bar{C} \in p(R / I)$. Hence $\pi^{-1}(\bar{C}) \in p(R)$ by 2.11 where $\pi$ is the canonical mapping of $R$ onto $R / I$ and $\pi^{-1}(\bar{C})$ is a two sided ideal. Thus $\bar{C}$ would be a two sided ideal in $R / I$. This proves that $R / I$ is a right Ore domain. Thus $I$ is an almost maximal right ideal.

4. If $R$ is a commutative ring then $R$ is a field if and only if every ideal which is not $R$ is a prime ideal. In this section, we will prove that $R$ is a simple ring if and only if every right ideal, which is not $R$, is of a prime type. We will also show that if $R$ is a simple ring then $\operatorname{dim}_{r} R=n$ if and only if $R=D_{n+1}$ for some division ring $D$.

4.1. LeMMA. If every right ideal of a ring $R$ is either of a prime type or $R$, then $R$ is semisimple.

Proof. Let $J(R)$ be the Jacobson radical of $R$ and assume $J(R) \neq 0$. Let $x$ be a nonzero element of $J(R)$. Let $I(x)$ be a right ideal which is maximal with respect to a property that $x \notin I(x)$. Then the right $R$-module $(x R+I(x) / I(x))$ is a simple $R$-module. Hence 
$(x R+I(x)) J(R) \subseteq I(x)$. Since $I(x) \neq R$, by hypothesis $I(x)$ is of a prime type. Thus $R J(R) \subseteq I(x)$ and $x \in J(R) \subseteq I(x)$. This is impossible since $x \notin I(x)$.

4.2. THEOREM. $R$ is a simple ring if and only if every right ideal of $R$ is either of a prime type or $R$.

Proof. Assume $R$ is a simple ring. Let $I$ be a right ideal of $R$ such that $I \neq R$. If $A B \subseteq I$ and $B \neq 0$ then $A=A R=A R B \subseteq A B \subseteq I$. Therefore, $I \in p(R)$. Assume that every proper right ideal of $R$ is of a prime type. Let $S$ be a two sided ideal such that $S \neq R$. Let $I$ be a maximal right ideal. If $S \nsubseteq I$ then $S \cap I$ is not of a prime type. For if $S \cap I$ is of a prime type then $I S \subseteq S \cap I$ implies that either $I \subseteq S \cap I$ or $S \subseteq S \cap I$. In any case, this means that $S \subset I$. Since $S \cap I \neq R$, a supposition that $S \nsubseteq I$ is absurd. Therefore $S \subseteq J(R)$. Since $J(R)=0$ by 4.1 , this means that $S=0$.

4.3. Theorem. Let $R$ be a (right) primitive ring. Then the following statements are equivalent:

(i) $\operatorname{dim}_{r} R=n$.

(ii) $R$ is isomorphic to the $n+1 \times n+1$ matrix ring over a division ring.

Proof. Let $M$ be a faithful simple $R$-module. If $\Gamma$ is a nonempty subset of $M$ then either $\bigcap_{m \in \Gamma}(m)^{\perp}=R$ or $\bigcap_{m \in \Gamma}(m)^{\perp} \in p(R)$ by 3.2. Hence if $\operatorname{dim}_{r} R=n$ then a length of any chain of the form $\left(m_{1}\right)^{\perp}$ $\supset\left(m_{1}\right)^{\perp} \cap\left(m_{2}\right)^{\perp} \supset \cdots \supset\left(m_{1}\right)^{\perp} \cap\left(m_{2}\right)^{\perp} \cdots \cap\left(m_{t}\right)^{\perp}$ for $m_{i} \in M, i=1$, $2, \cdots$, must be less than or equal to $n$. This means that the vector space dimension of $M$ over its endomorphism ring is less than or equal to $n+1$. Thus $R$ is a simple ring with the minimum condition on right ideals and $R$ is a direct sum of $m$ minimal right ideals. Since, by 4.2 , every proper right ideal of $R$ is of a prime type and since $\operatorname{dim}_{r} R=n, m \leqq n+1$. Let $(0)=P_{0} \subset P_{1} \subset \cdots \subset P_{n}$ be a chain of $n$-distinct right ideals of a prime type. Since every right ideal of a simple right artinian ring is a direct summand of $R$, there exist a sequence of nonzero right ideals $K_{0}, K_{1}, \cdots, K_{n}$ such that $P_{0} \oplus K_{0}=P_{1}, P_{1} \oplus K_{1}=P_{2}, \cdots, P_{n-1} \oplus K_{n-1}=P_{n}$ and $P_{n} \oplus K_{n}=R$. Hence $R=P_{0} \oplus K_{1} \oplus K_{2} \oplus \cdots K_{n}=K_{1} \oplus K_{2} \oplus \cdots \oplus K_{n}$. Since the number of direct summands in a decomposition of $R$ as a direct sum of minimal right ideals in $R$ is unique, $n+1 \leqq m$. Thus $n+1=m$ and $R$ is isomorphic to $D_{n+1}$ for some division ring $D$. Conversely, if $R$ is isomorphic to the $n+1 \times n+1$ matrix ring over a division ring then $R$ is a simple right artinian ring and $R$ is a direct sum of $n+1$ minimal right ideals, say $I_{1}, I_{2}, \cdots, I_{n}$. The length of chain (0) $=I_{0} \subset I_{1} \subset I_{1}+I_{2} \subset \cdots \subset I_{1}+I_{2}+\cdots+I_{n}$ is $n$ and each com- 
ponent of the chain is a right ideal of a prime type by 4.2. Furthermore no chain of right ideals of prime type can exceed $n$ by an argument given above in a proof of the necessity. Thus $\operatorname{dim}_{r} R=n$.

EXAMPLE 4.4. A noncommutative regular ring can have an infinite dimension. For example, let $R$ be the ring of all linear transformations of infinite-dimensional vector space over a division ring $D$. Then $R$ is a regular ring which is primitive (refer $[5$, p. 123]). Hence by $4.3, \operatorname{dim}_{r} R$ cannot be finite.

EXAMPLE 4.5. If $R$ is a commutative ring then the concepts of almost maximal right ideal, a right ideal of a prime type and prime ideal are all the same. However, in a noncommutative ring they are quite different. In fact, in any right artinian ring the supremum of the lengths of all chains of almost maximal right ideals is exactly zero by 3.4 of [5]. However $\operatorname{dim}_{r} R$ could be an arbitrary finite number since any $n \times n$ matrix ring over a division ring is an artinian ring which has the dimension $n-1$.

EXAmple 4.6. Let $Z$ be the ring of integers. Then $\operatorname{dim} Z=1$. Let $R=\left(\begin{array}{l}Z Z \\ 0 Z\end{array}\right)$, the $2 \times 2$ upper triangular matrix ring over $Z$. Then $\operatorname{dim}_{r} R=1$ since $\operatorname{rad} R=\left(\begin{array}{ll}0 Z \\ 0 & Z\end{array}\right)$ and $R / \operatorname{rad} R \cong Z \times Z$. However $R$ cannot be isomorphic to a $2 \times 2$ matrix ring over any ring.

EXAMPLE 4.7. If $R$ is a commutative principal ideal domain then $\operatorname{dim}_{r} R=1$. However, there exists a principal right and left ideal domain whose right dimension is infinite. For example, let $F(\xi)$ be the ring of rational functions in $\xi$ over a field $F$ of zero characteristic and take $F[x, \xi]$ to be the ring of differential polynomials in $t$ over $F(\xi)$. Its elements are subject to the rule $\alpha t=t \alpha+d \alpha / d \xi, \alpha \in F(\xi)$. Then $F[x, \xi]$ is a simple principal right and left domain which is not a division ring (refer [2]). Hence by 4.3 , the dimension of $F[x, \xi]$ can not be finite.

Acknowledgement. The author is indebted to the referee for many helpful suggestions.

\section{REFERENCES}

1. C. Faith, Rings with ascending conditions on annihilators, Nagoya Math. J. 27 (1966), 179-191. MR 33 \#1328.

2. N. Jacobson, Structure of rings, 2nd rev. ed., Amer. Math. Soc. Colloq. Publ., vol. 37, Amer. Math. Soc., Providence, R. I., 1964. MR 36 \#5158.

3. K. Koh, On almost maximal right ideals, Proc. Amer. Math. Soc. 25 (1970), 266-272.

4. - On some characteristic properties of self-injective rings, Proc. Amer. Math. Soc. 19 (1968), 209-213. MR 36 \#1485.

5. N. H. McCoy, The theory of rings, Macmillan, New York, 1964. MR 32 \#5680.

North Carolina State University, Raleigh, North Carolina 27607 